ARTICLE

https://doi.org/10.1038/s41467-019-11289-4

\title{
Targeting metabolic driving and intermediate influx in lysine catabolism for high-level glutarate production
}

Wenna Li ${ }^{1}$, Lin Ma ${ }^{1}$, Xiaolin Shen ${ }^{1}$, Jia Wang ${ }^{1}$, Qi Feng ${ }^{1}$, Lexuan Liu ${ }^{1}$, Guojun Zheng ${ }^{1}$, Yajun Yan², Xinxiao Sun (D) ${ }^{1} \&$ Qipeng Yuan ${ }^{1}$

Various biosynthetic pathways have been designed to explore sustainable production of glutarate, an attractive $\mathrm{C} 5$ building block of polyesters and polyamides. However, its efficient production has not been achieved in Escherichia coli. Here, we use E. coli native lysine catabolic machinery for glutarate biosynthesis. This endogenous genes-only design can generate strong metabolic driving force to maximize carbon flux toward glutarate biosynthesis by replenishing glutamate and $\mathrm{NAD}(\mathrm{P}) \mathrm{H}$ for lysine biosynthesis, releasing lysine feedback inhibition, and boosting oxaloacetate supply. We use native transporters to overcome extracellular accumulation of cadaverine and 5 -aminovalerate. With these efforts, both high titer $\left(54.5 \mathrm{~g} \mathrm{~L}^{-1}\right)$ and high yield $\left(0.54 \mathrm{~mol} \mathrm{~mol}^{-1}\right.$ glucose) of glutarate production are achieved under fed-batch conditions. This work demonstrates the power of redirecting carbon flux and the role of transporters to decrease intermediate accumulation.

\footnotetext{
${ }^{1}$ State Key Laboratory of Chemical Resource Engineering, Beijing University of Chemical Technology, Beijing 100029, China. ${ }^{2}$ School of Chemical, Materials and Biomedical Engineering, College of Engineering, The University of Georgia, Athens, GA 30602, USA. Correspondence and requests for materials should be addressed to X.S. (email: sunxx@mail.buct.edu.cn) or to Q.Y. (email: yuanqp@mail.buct.edu.cn)
} 
$\mathrm{D}$ icarboxylic acids are important building blocks for manufacturing polyesters and polyamides. Due to the concerns on oil supply and environment protection, increasing attention has been attracted to developing green and sustainable routes to these chemicals. Biosynthesis represents a promising alternative. Currently, bio-production of succinate is mature enough for commercial application and the titer reaches $152.2 \mathrm{~g} \mathrm{~L}^{-1}$ from glucose by recombinant Corynebacterium glutamicum $^{1}$. Recently, production of adipic acid with high titer (68 $\left.\mathrm{g} \mathrm{L}^{-1}\right)$ and yield $\left(0.38 \mathrm{~g} \mathrm{~g}^{-1}\right)$ has been achieved via a reversal $\beta$ oxidation pathway ${ }^{2}$. Glutarate is another important dicarboxylic acid used to manufacture polymers such as nylon- 4,5 and nylon5 , 5. The lowest melting point of glutarate among aliphatic dicarboxylic acids may endow the resultant polymers with unique properties. In addition, glutarate is a synthetic precursor of 1, 5pentanediol, a common plasticizer and a precursor of polyesters ${ }^{3}$.

Over the years, several pathways have been designed for glutarate biosynthesis via 2-oxogularate reduction ${ }^{4}$, reverse $\beta$ oxidation from acetyl-CoA and malonyl- $\mathrm{CoA}^{5}$, carbon chain extension and decarboxylation from 2-oxogularate ${ }^{6}$, and lysine catabolism $^{4,7}$. Among them, the lysine catabolism routes have the highest reported titers. Lysine can be degraded to glutarate via two partially different pathways, 5-aminovalerate (AMV) pathway and Cadaverine pathway ${ }^{8,9}$ (Fig. 1a). In AMV pathway, lysine is converted to AMV by the sequential catalysis of lysine 2monooxygenase and delta-aminovaleramidase. AMV is further converted to glutarate by 5 -aminovalerate transaminase and glutarate semialdehyde dehydrogenase. In Cadaverine pathway, conversion of lysine to AMV is catalyzed alternatively by lysine decarboxylase, cadaverine aminotransferase, and aminovaleraldehyde dehydrogenase. C. glutamicum has been extensively investigated for lysine production ${ }^{10,11}$. Recently, both AMV and Cadaverine pathways have been constructed in lysineoverproducing C. glutamicum strains $s^{4,7,12}$. The highest reported titer reached $90 \mathrm{~g} \mathrm{~L}^{-1}$ with the yield to be $0.28 \mathrm{~mol} \mathrm{~mol}^{-1}$ in the growth phase and $0.70 \mathrm{~mol} \mathrm{~mol}^{-1}$ in the production phase, respectively ${ }^{12}$. E. coli is another commonly used host for metabolic engineering. However, reconstructing AMV pathway in a lysine-overproducing E. coli strain resulted in only $0.82 \mathrm{~g} \mathrm{~L}^{-1}$ of glutarate in shake flasks, representing $9.1 \%$ of the theoretical yield $\left(0.75 \mathrm{~mol} \mathrm{~mol}^{-1} \text { glucose }\right)^{13}$. The possible reasons are that (1) the pathways have unresolved limiting factors preventing carbon flux from reaching the end product and (2) the introduction of the downstream pathway perturbs the existing metabolic balance.

In fact, $E$. coli contains a native Cadaverine pathway, which is regulated by catabolite repression and induced by nitrogen limitation $^{14,15}$. In this study we aim to engineer $E$. coli into an efficient glutarate producer by modulating its own genes. From oxaloacetate the full pathway involves 15 enzymatic steps (Fig. 1b), which represents a great challenge to achieve enhanced and balanced expression. Driving forces are essential for efficient biosynthesis of a target compound. They can be present as a push (e.g., increasing precursor supply), a pull (e.g., efflux or removal of the end product) or an internal recycle pump (e.g., redox balance). Under anaerobic conditions, NADH is frequently used as an internal recycle pump for the production of lactate, succinate, ethanol, and 1-butanol ${ }^{16-19}$. Under aerobic conditions, overexpressing key pathway enzymes and product exporters are usually used as the driving forces ${ }^{20}$. However, for long pathways, overexpression of multiple genes often causes metabolic burden and requires careful balance via strategies like multivariate modular optimization ${ }^{21,22}$. As indicated by previous studies, boosting lysine supply is not guaranteed for efficient glutarate production.

After analyzing the glutarate biosynthetic pathway, we find that at least three different driving forces (recycle of glutamate, recycle of redox power, and release of feedback inhibition) can be harnessed to continuously drag carbon flux through the target pathway. E. coli Cadaverine pathway involves one decarboxylation (CadA), two transamination (PatA and GabT), and two dehydrogenation reactions (PatD and GabD) (Fig. 1). Although it is one-step longer than AMV pathway, Cadaverine pathway requires no participation of molecular oxygen, which is advantageous for large-scale cultivation. Furthermore, per lysine consumed, the transamination reactions generate two glutamate and the dehydrogenases produce two $\mathrm{NAD}(\mathrm{P}) \mathrm{H}$, respectively (Fig. 1). Interestingly, starting from oxaloacetate, the lysine biosynthetic pathway consumes two glutamate and two $\mathrm{NAD}(\mathrm{P}) \mathrm{H}$ to produce one lysine. Therefore, lysine catabolism could replenish glutamate and $\mathrm{NAD}(\mathrm{P}) \mathrm{H}$ for lysine biosynthesis. In addition, lysine consumption could naturally release its feedback inhibition on upstream genes and enzymes, representing as another driving force.

As expected, the synergistic use of these driving forces significantly improves production efficiency. In addition, extracellular accumulation of two pathway intermediates is observed and the problem is solved by identifying and expressing specific transporters. Further boosting oxaloacetate supply by activating glyoxylate cycle generates a final strain that produces glutarate with the highest reported titer and yield in shake flasks. This study provides a convenient and effective strategy for metabolic engineering of other long pathways.

\section{Results}

Pathway assembly using endogenous enzymes. E. coli native genes cadA, patAD, and gabTD were used to construct the glutarate biosynthetic pathway. Gene cadA encodes an inducible lysine decarboxylase. Compared with its constitutive counterpart LdcC, CadA shows higher activity and thermal stability ${ }^{23}$. PatA and PatD are annotated as putrescine aminotransferase and $\gamma$ aminobutyraldehyde dehydrogenase, and GabT and GabD as 4aminobutyrate aminotransferase and succinate-semialdehyde dehydrogenase, respectively. These four enzymes show promiscuous activity and are involved in putrescine and cadaverine catabolism $^{4,24,25}$.

Based on the functional relevance, these genes were separated into three modules (cadA, patAD, and gabTD) and their expression was modulated stepwise using plasmids with different copy numbers. The results of feeding experiments showed that the low- and medium-copy plasmids (pSA-cadA and pCS-cadA) are more suitable for carrying cadA module than the high-copy plasmid (pZE-cadA). Strains BW (pSA-cadA) and BW (pCScadA) effectively converted lysine to cadaverine with the titers of $2.62 \pm 0.07$ (mean $\pm \mathrm{SD}$, same for the following) $\mathrm{g} \mathrm{L}^{-1}$ and $2.57 \pm$ $0.13 \mathrm{~g} \mathrm{~L}^{-1}$, respectively while the titer by strain $\mathrm{BW}$ (pZE-cadA) was only $0.35 \pm 0.08 \mathrm{~g} \mathrm{~L}^{-1}$ (Fig. 2a). The control strain also produced tiny amount of cadaverine $\left(0.13 \pm 0.01 \mathrm{~g} \mathrm{~L}^{-1}\right)$ due to the basal activity of the lysine decarboxylases (Fig. 2a).

Similarly, patAD module was optimized using cadaverine as the substrate. However, no obvious conversion occurred with all the three strains. To check the activity of PatA and PatD, $6 \times$ Histagged proteins were purified and the result of in vitro assays showed that both enzymes were functionally expressed and the specific activities were $84.40 \mathrm{~min}^{-1}$ and $11.99 \mathrm{~min}^{-1}$, respectively. Accordingly, we speculated that the conversion is hindered by inefficient transport of cadaverine across the cell membrane. To verify this, patAD module was co-expressed with the optimized cadA module and lysine was used as the feeding substrate instead of cadaverine. The resultant strains successfully produced AMV. Strain BM23 carrying pSA-cadA and pCS-patAD produced the highest amount of AMV $\left(1.93 \pm 0.04 \mathrm{~g} \mathrm{~L}^{-1}\right)$ at $48 \mathrm{~h}$; notably, 
a
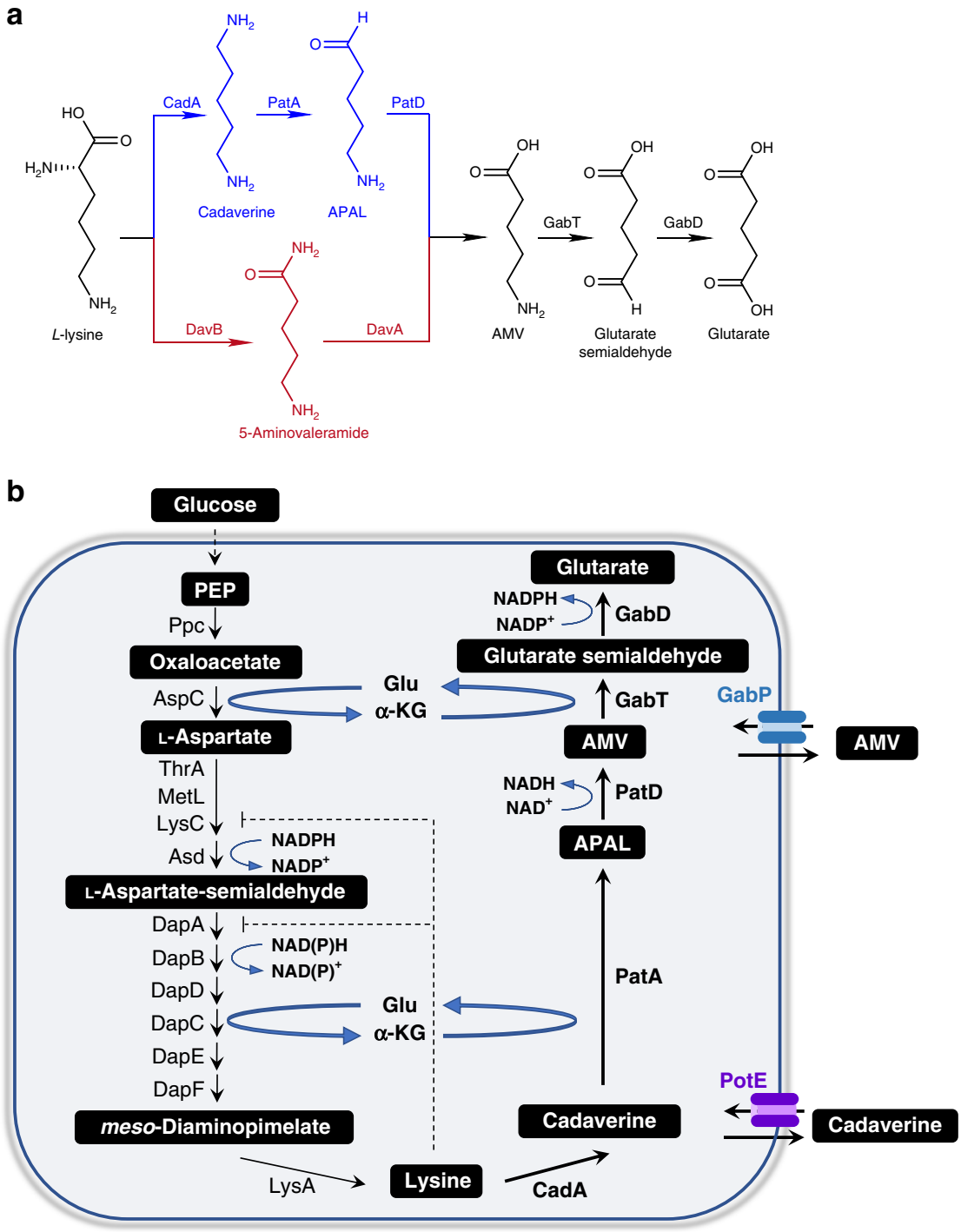

Fig. 1 Glutarate biosynthesis via lysine catabolism. a The Cadaverine pathway (blue color) and the AMV pathway (red color) from lysine to glutarate. b $E$. coli native pathway for glutarate biosynthesis. Thick arrows indicate gene overexpression. Enzymes: CadA, lysine decarboxylase; DavA, deltaaminovaleramidase; DavB, lysine 2-monooxygenase; GabD, succinate-semialdehyde dehydrogenase; GabT, 4-aminobutyrate-2-oxoglutarate transaminase; PatA, putrescine aminotransferase; PatD, aminobutyraldehyde dehydrogenase. Metabolites: Glu, glutamate; $\alpha-K G, \alpha-k e t o g l u t a r a t e ; ~ P E P$,

phosphoenolpyruvate; APAL, 5-aminopentanal; AMV, 5-aminovalerate

strain BM21 carrying pSA-patAD-cadA already accumulated as high as $0.77 \pm 0.05 \mathrm{~g} \mathrm{~L}^{-1}$ of glutarate (Fig. 2b).

When optimizing gabTD module, we observed a similar phenomenon as patAD module. When fed with $5 \mathrm{~g} \mathrm{~L}^{-1}$ of AMV, the strain carrying pSA-gabTD performed best but produced only $0.63 \pm 0.03 \mathrm{~g} \mathrm{~L}^{-1}$ of glutarate, even lower than that produced by strain BM21 (0.77 $\mathrm{g} \mathrm{L}^{-1}$ ) (Fig. 2c). Module gabTD was then grafted to the optimized cadA and patAD modules, resulting in two strains BM31(BW/pSA-cadA and pCS-patAD-gabTD) and BM32 (BW/ pSA-cadA-gabTD and pCS-patAD). Slightly better than BM32, strain $\mathrm{BM} 31$ produced $1.32 \pm 0.03 \mathrm{~g} \mathrm{~L}^{-1}$ of glutarate at $72 \mathrm{~h}$ in the feeding experiment. Furthermore, adding $2 \mathrm{~g} \mathrm{~L}^{-1}$ of a-ketoglutarate ( $\alpha-\mathrm{KG}$ ) improved the titer to $1.90 \pm 0.06 \mathrm{~g} \mathrm{~L}^{-1}$, suggesting that $\alpha$ KG supplement is beneficial to the transamination reactions (Fig. 2d). In addition, we observed extracellular accumulation of both cadaverine $\left(1.66 \pm 0.04 \mathrm{~g} \mathrm{~L}^{-1}\right.$ at $\left.72 \mathrm{~h}\right)$ and AMV $(1.19 \pm 0.08 \mathrm{~g}$ $\mathrm{L}^{-1}$ at $72 \mathrm{~h}$ ) (Fig. 3a, b), indicating that the re-uptake of these intermediates is rate-limiting. To solve this problem, specific transporters were screened and expressed in the next steps.
GabP can efficiently import AMV. In E. coli chromosome, gabP is clustered with $g a b T$ and $g a b D$ into an operon. GabP is annotated as a 4-aminobutyrate transporter. Considering the promiscuous role of GabT and GabD in degradation of cadeverine and putrescine ${ }^{4,24}$, we speculate that GabP may also be able to transport AMV.

To explore the potential of GabP for AMV importation, strain BM31P (BW/pSA-cadA and pCS-patAD-gabTDP) was constructed and used for the feeding experiment. The result showed that extracellular amount of AMV first increased to $1.30 \pm 0.01 \mathrm{~g}$ $\mathrm{L}^{-1}$ at $24 \mathrm{~h}$ and thereafter decreased significantly to nondetectable at $72 \mathrm{~h}$, while the control strain BM31 accumulated $1.56 \pm 0.04 \mathrm{~g} \mathrm{~L}^{-1}$ of AMV at $24 \mathrm{~h}$ and the amount decreased slightly to $1.19 \pm 0.08 \mathrm{~g} \mathrm{~L}^{-1}$ at $72 \mathrm{~h}$ (Fig. 3a). The result indicates that GabP indeed is a functional AMV transporter and increasing its expression can reduce extracellular accumulation of AMV. Consequently, glutarate titer was significantly improved from $1.36 \pm 0.03 \mathrm{~g} \mathrm{~L}^{-1}$ to $2.34 \pm 0.01 \mathrm{~g} \mathrm{~L}^{-1}$ (Fig. 3a). Recently, it was shown that expression of an importer is also crucial for efficient 

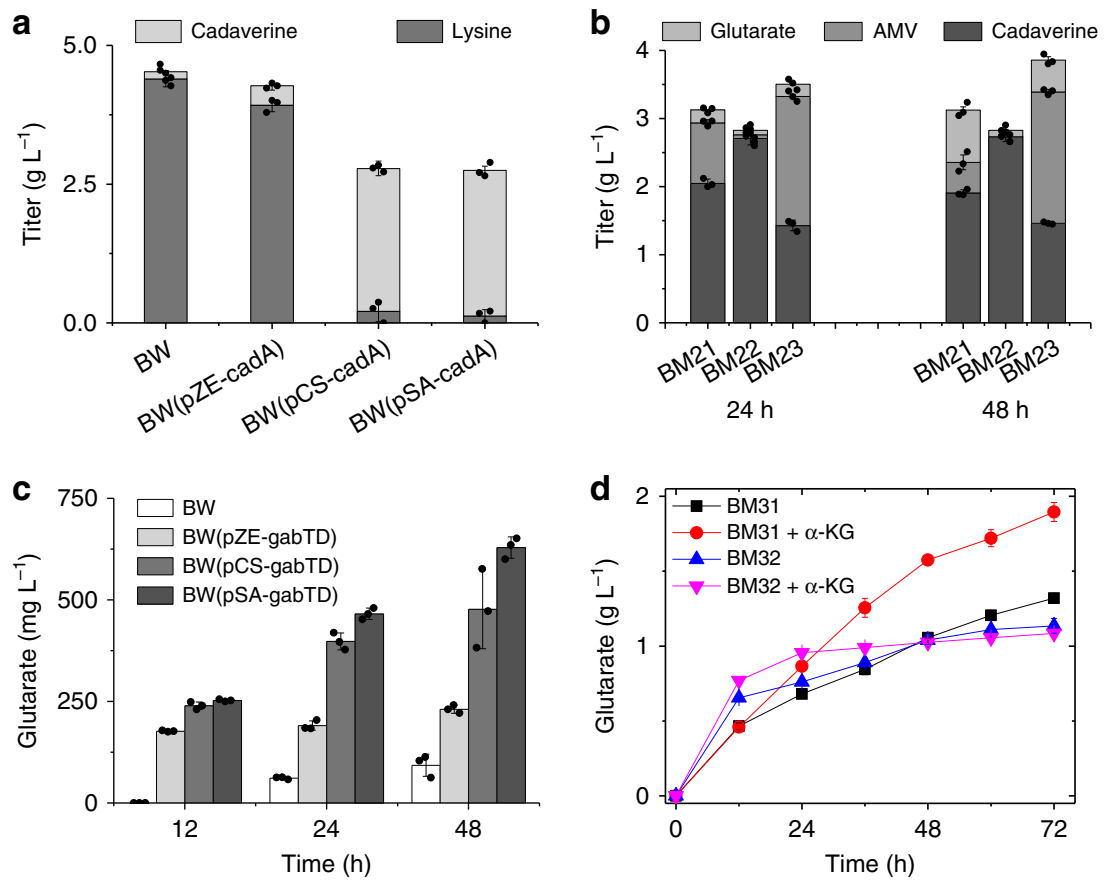

Fig. 2 Modular optimization of glutarate production from lysine. Optimization of a cadA module, $\mathbf{b}$ patAD module, and $\mathbf{c}$ gabTD module. $\mathbf{d}$ Glutarate production from lysine. $\alpha-K G, \alpha-k$ etoglutarate. Data shown are mean \pm SD ( $n=3$ independent experiments). Source data are provided as a Source Data file
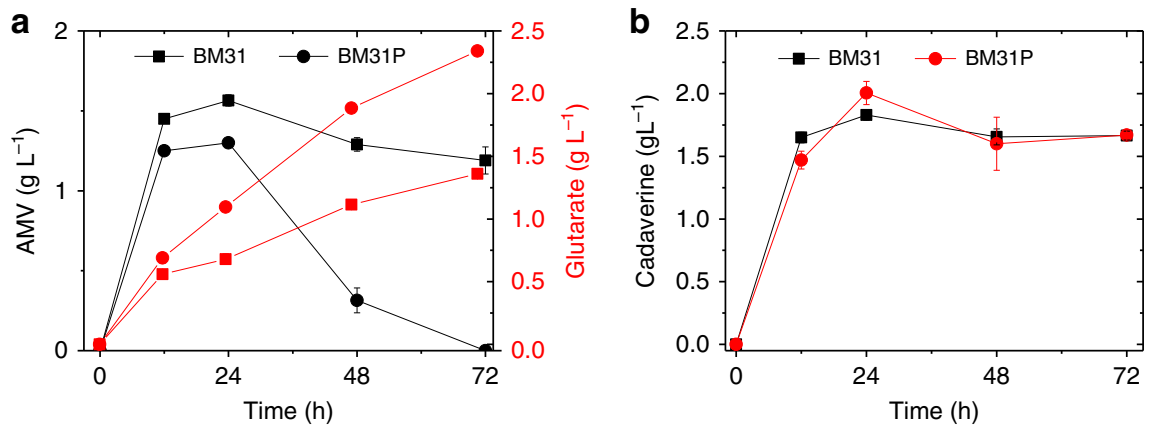

Fig. 3 Overexpression of gabP decreases AMV accumulation. Effect of gabP expression on the accumulation of a AMV and glutarate, and $\mathbf{b}$ cadaverine. Data shown are mean \pm SD ( $n=3$ independent experiments). Source data are provided as a Source Data file

re-uptake of 5-aminovalerate in C. glutamicum. ${ }^{12}$ However, the problem of cadaverine accumulation remained unsolved (Fig. 3b).

PotE is a bi-directional cadaverine transporter. Theoretically, extracellular accumulation of cadaverine can be decreased by either preventing its efflux or enhancing its uptake. However, it seems challenging to implement the first strategy, because cadaverine can be exported via multiple potential exporters/antiporters including $\mathrm{CadB}$, SapB, PotE, and yet un-characterized ones. $\mathrm{CadB}$ is both cadaverine importer and a lysine:cadaverine antiporter ${ }^{26}$, SapB is a subunit of the newly characterized putrescine exporter SapBCDF ${ }^{27}$, and PotE is a bi-directional putrescine transporter ${ }^{28}$. To validate their effect on cadaverine export, three single knockout strains (BW $\Delta c a d B$, BW $\Delta s a p B$, and BW $\Delta$ potE) were transformed with plasmids pSA-cadA and pCSpatAD-gabTDP, and the resultant strains were fed with $5 \mathrm{~g} \mathrm{~L}^{-1}$ of lysine. The results showed that the single knockouts had no positive effect on glutarate production and knocking out potE or $s a p B$ even caused impaired cell growth and decreased glutarate production (Supplementary Fig. 1). Thus, the performance of multiple knockouts was not further tested and the second strategy was adopted instead.

E. coli contains multiple importers including PotE, PuuP, PotABCD, and PotFGHI ${ }^{28-31}$. However, their capabilities of transporting cadaverine have not been investigated before. Their encoding genes were cloned into plasmid pSA74. Co-transferring the resultant plasmids with pCS-patAD into strain BW25113 (BW, for short) generated four corresponding strains (BW2E, BW2P, BW2A, and BW2F), which were used for the feeding experiment. Among the four importers, PotE was shown to be the most effective and strain BM2E containing pSA-potE and pCSpatAD completely transported and converted $1 \mathrm{~g} \mathrm{~L}^{-1}$ of cadaverine into AMV within $12 \mathrm{~h}$ (Fig. 4a, b).

In order to further verify the function of PotE, strain BM31PE was constructed and cultured in M9 medium supplemented with $5 \mathrm{~g} \mathrm{~L}^{-1}$ of lysine. Compared with that of strain BM31P (1.60 \pm $\left.0.07 \mathrm{~g} \mathrm{~L}^{-1}\right)$, strain BM31PE accumulated higher amount of extracellular cadaverine $\left(2.04 \pm 0.04 \mathrm{~g} \mathrm{~L}^{-1}\right)$ at $12 \mathrm{~h}$ and then the amount decreased to $1.53 \pm 0.01 \mathrm{~g} \mathrm{~L}^{-1}$ at $72 \mathrm{~h}$, which is comparable to that of BM31P (1.56 $\left.\pm 0.11 \mathrm{~g} \mathrm{~L}^{-1}\right)$ (Supplementary Fig. 2). This result confirmed that PotE is a bi-directional cadaverine 

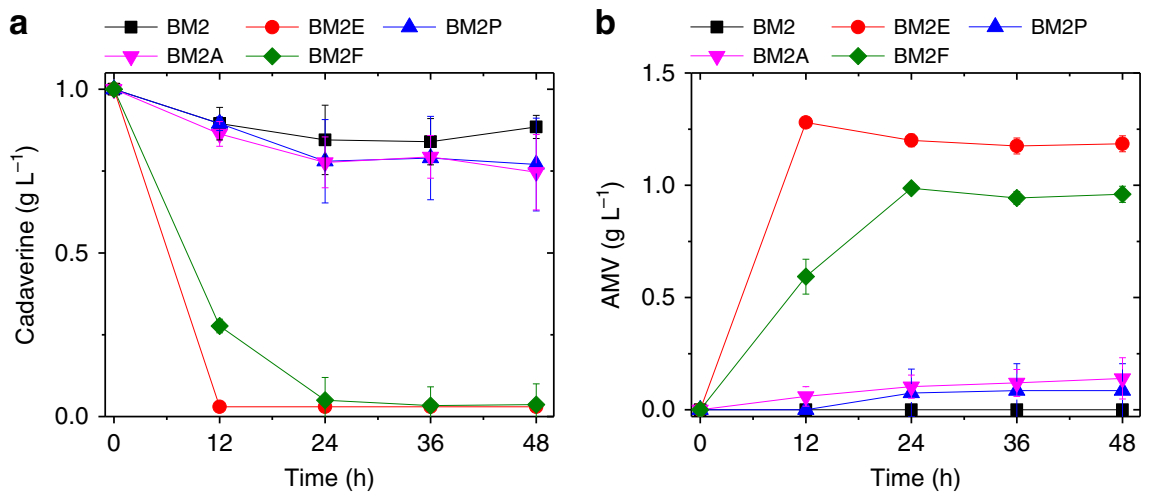

Fig. 4 Comparison of the efficiency of four candidate cadaverine transporters. a Cadaverine consumption with time; b AMV accumulation with time. Data shown are mean \pm SD ( $n=3$ independent experiments). Source data are provided as a Source Data file
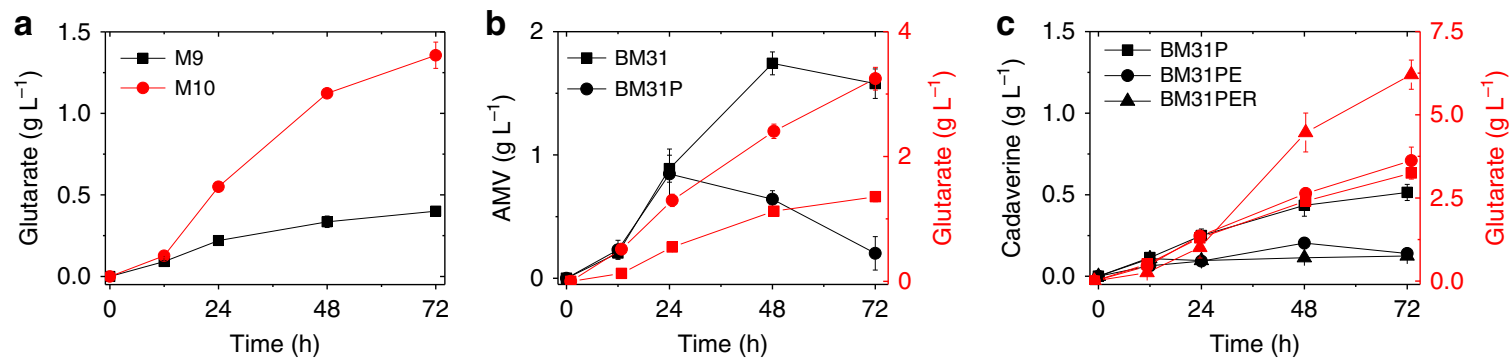

Fig. 5 De novo biosynthesis of glutarate. a Comparison of glutarate production in M9 and M10 media. b Comparison of glutarate production and AMV accumulation by strains BM31 and BM31P. c Comparison of glutarate production and cadaverine accumulation by strains BM31P, BM31PE, and BM31PER. Data shown are mean \pm SD ( $n=3$ independent experiments). Source data are provided as a Source Data file

transporter. Under this condition, cadaverine is prone to be exported to decrease its intracellular accumulation.

De novo biosynthesis of glutarate. To explore de novo biosynthesis of glutarate, strains BW and BM31 were cultivated in M9 medium with $10 \mathrm{~g} \mathrm{~L}^{-1}$ of glucose. E. coli BW produced nondetectable lysine and glutarate, while strain BM31 produced $0.40 \mathrm{~g} \mathrm{~L}^{-1}$ of glutarate at $72 \mathrm{~h}$ (Fig. 5a). This demonstrates thatthe enhancement of lysine catabolism generates expected driving forces. Glutarate biosynthesis involves multiple transamination reactions and the ammonia-rich M10 medium was used previously for glutarate production in $E$. colitis. Culturing strain BM31 in M10 medium increased glutarate titer to $1.36 \pm$ $0.08 \mathrm{~g} \mathrm{~L}^{-1}$ (Fig. 5a). Meanwhile, $1.58 \pm 0.12 \mathrm{~g} \mathrm{~L}^{-1}$ of AMV and $0.32 \pm 0.03 \mathrm{~g} \mathrm{~L}^{-1}$ of cadaverine were accumulated (Supplementary Fig. 3). By contrast, strain BM31P produced $3.25 \pm 0.18 \mathrm{~g} \mathrm{~L}^{-1}$ of glutarate, while extracellular AMV decreased to $0.20 \pm 0.14 \mathrm{~g} \mathrm{~L}^{-1}$ (Fig. 5b), confirming that AMV influx is crucial for efficient glutarate production. Unlike in the feeding experiment, in de novo production overexpression of PotE showed positive effect on cadaverine importation, leading to a slight increase in glutarate titer $\left(3.62 \pm 0.28 \mathrm{~g} \mathrm{~L}^{-1}\right)$ by strain BM31PE (Fig. $5 \mathrm{c}$ ).

Boosting oxaloacetate supply enhances glutarate production. With the above efforts, glutarate production was improved stepwise. However, glutarate yield $\left(0.20 \mathrm{~mol} \mathrm{~mol}^{-1}\right)$ by strain BM31PE is still far below the theoretical value. We then attempted to enhance glutarate production by overexpressing key upstream genes including phosphoenolpyruvate carboxylase, aspartate kinase, and 4-hydroxy-tetrahydrodipicolinate synthase, which has been shown to be effective in increasing production of lysine and its derivatives ${ }^{32-34}$. However, the resultant strains produced less amount of glutarate than strain BM31PE
(Supplementary Fig. 4), suggesting that the prior balance may be disturbed. Instead of modulating expression of these genes, we turned to enhance oxaloacetate supply by deleting $i c l R$. IclR is a transcription factor that represses expression of the glyoxylate bypass operon ${ }^{34}$. Glyoxylate bypass is a common mechanism of replenishing oxaloacetate consumed for biosynthesis. Strikingly, deletion of $i c l R$ led to a significant increase in glutarate production. The titer by strain BM31PER reached $6.21 \pm 0.44 \mathrm{~g} \mathrm{~L}^{-1}$ $\left(0.34 \mathrm{~mol} \mathrm{~mol}^{-1}\right)$ at $72 \mathrm{~h}$. The result demonstrates that boosting oxaloacetate supply provided another strong driving force.

Confirming the importance of driving forces. To further demonstrate the importance of driving forces, we constructed three strains BWL0 (BW $\Delta c a d A \Delta l d c C)$, BWL1 (BW $\Delta p a t A / p S A-$ cadA), and BWL3 (BW $\Delta g a b T / p S A-c a d A$ and pCS-patAD) to produce lysine, cadaverine, and AMV, respectively. As expected, the titers of the corresponding products increased with the extension of the pathway (Table 1). Under normal conditions, lysine biosynthesis is tightly regulated. Strain BWL0 produced non-detectable lysine because of the lack of driving forces. Strain BWL1 produced $1.52 \pm 0.05 \mathrm{~g} \mathrm{~L}^{-1}$ of cadaverine, indicating that converting lysine to cadaverine creates driving force by releasing feedback inhibition on upstream genes and enzymes. Furthermore, strain BWL3 produced $3.44 \pm 0.15 \mathrm{~g} \mathrm{~L}^{-1}$ of AMV, confirming that the transamination and dehydrogenation steps provided additional driving forces. Further extending the pathway resulted in a minor increase glutarate titer $\left(3.62 \pm 0.28 \mathrm{~g} \mathrm{~L}^{-1}\right)$ by BM31PE, suggesting that there may be a compromise between the pathway length and the driving force strength. In addition, two control strains (BWL5/C1 and C2) were also constructed. As noticed above, knocking out potE caused unhealthy cell growth. Therefore, strain BWL5/C1 with potE and $g a b P$ double knockouts showed both decreased glutarate production and increased AMV 
Table 1 Verification of the effect of driving forces on the pathway efficiency

\begin{tabular}{|c|c|c|c|c|c|}
\hline Strains & Description & Lysine $\left(\mathrm{g} \mathrm{L}^{-1}\right)$ & Cadaverine $\left(\mathrm{g} \mathrm{L}^{-1}\right)$ & $A M V\left(g^{-1}\right)$ & Glutarate $\left(\mathrm{g} \mathrm{L}^{-1}\right)$ \\
\hline BWLO & $\mathrm{BW} \Delta \mathrm{cad} A \Delta / d c \mathrm{C}$ & $N^{*}$ & ND & ND & ND \\
\hline BWL1 & $\mathrm{BW} \Delta$ patA/pSA-cadA & ND & $1.52 \pm 0.05^{\star \star}$ & $0.17 \pm 0.02$ & ND \\
\hline BWL3 & BW $\Delta g a b T / p S A-c a d A, p C S-p a t A D$ & ND & $0.67 \pm 0.12$ & $3.44 \pm 0.15$ & ND \\
\hline BM31PER & BW $\Delta$ iclR/pSA-cadA-potE, pCS-patAD-gabTDP & ND & $0.12 \pm 0.02$ & $0.32 \pm 0.11$ & $6.21 \pm 0.44$ \\
\hline BWL5/C1 & BW $\Delta$ gabP $\Delta$ potE/pSA-cadA, pCS-patAD-gabTD & ND & ND & $1.44 \pm 0.08$ & $0.73 \pm 0.12$ \\
\hline BWL5/C2 & BW/pCS-patAD-gabTDP & ND & ND & ND & $0.91 \pm 0.12$ \\
\hline
\end{tabular}
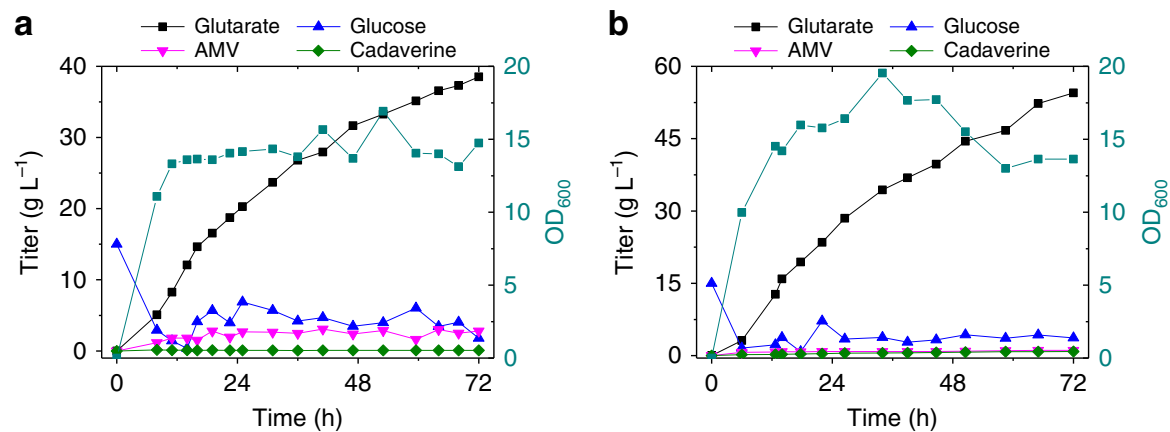

Fig. 6 Fed-batch production of glutarate in $3 \mathrm{~L}$ bioreactors. Strains BM31PE (a) and BM31PER (b) were used for the experiments, respectively. The minimum threshold of DO was set at $10 \%$. The result of a duplicate experiment is shown in Supplementary Fig. 6, which results in similar titer and yield. Source data are provided as a Source Data file

accumulation. Strain BWL5/C2 with the basal expression of CadA produced only $0.91 \pm 0.12 \mathrm{~g} \mathrm{~L}^{-1}$ of glutarate. These results indicate that the existence of limiting steps could weaken the effectiveness of the driving forces.

Fed-batch production of glutarate. To evaluate the scale-up potential of glutarate production, fed-batch experiments were carried out in bioreactors using strains BM31PE and BM31PER. We investigated the effect of dissolved oxygen (DO) on glutarate production. The results showed that high DO is benefit for cell growth but not for glutarate accumulation. When DO was set at $20 \%$, the cell density $\left(\mathrm{OD}_{600}\right)$ of strain $\mathrm{BM} 31 \mathrm{PE}$ reached 50 , while the final titer of glutarate was only $8 \mathrm{~g} \mathrm{~L}^{-1}$ from $137 \mathrm{~g} \mathrm{~L}^{-1}$ glucose (Supplementary Fig. 5). On the contrary, at $10 \%$ set DO, the cells entered the stationary phase within $12 \mathrm{~h}$ and the $\mathrm{OD}_{600}$ value fluctuated around 15 till the end of the cultivation (Fig. 6a). Interestingly, glutarate was produced continuously independent of cell growth, demonstrating the advantage of interior balance of the pathway. At $72 \mathrm{~h}, 38.5 \mathrm{~g} \mathrm{~L}^{-1}\left(0.48 \mathrm{~mol} \mathrm{~mol}^{-1}\right)$ of glutarate was produced with the consumption of $111 \mathrm{~g} \mathrm{~L}^{-1}$ of glucose. Both AMV and cadaverine were accumulated at low levels. Acetate was the main byproduct, which accumulated $16 \mathrm{~g} \mathrm{~L}^{-1}$ by the end of cultivation. When strain BM31PER was used, it produced $54.5 \mathrm{~g} \mathrm{~L}^{-1}$ of glutarate at $72 \mathrm{~h}$ and the yield $\left(0.54 \mathrm{~mol} \mathrm{~mol}^{-1}\right)$ reached $72 \%$ of the theoretical maximum (Fig. $6 \mathrm{~b}$ ).

\section{Discussion}

In order to adapt to the changing environment, microorganisms contain various degradation pathways, in which may exist some useful intermediates, e.g., muconic acid ${ }^{35,36}$ and maleic acid ${ }^{37}$ in aromatic compound degradation. Connecting a degradation pathway with the corresponding biosynthetic pathway can lead to de novo biosynthesis of the target compound. In E. coli, lysine decarboxylation to cadaverine is involved in its adaption to acid stress $^{38}$. Cadaverine can be re-assimilated into the TCA cycle via further enzymatic steps ${ }^{9}$. In this study, we used $E$. coli native lysine biosynthetic and catabolic mechanism for glutarate production without introducing foreign genes.

The long distance from the central metabolism means that only limited flux could be allocated to glutarate under normal conditions. In previous studies, lysine-overproducing strains are commonly used to ensure enough precursor supply. For example, lysine-hyperproducing strain C. glutamicum LYS-12 was used as the chassis strain. With 18 genomic changes the best strain produced $1.85 \mathrm{~g} \mathrm{~L}^{-1}\left(0.27 \mathrm{~mol} \mathrm{~mol}^{-1}\right)$ of glutarate in shake flasks and $90 \mathrm{~g} \mathrm{~L}^{-1}$ of glutarate under fed-batch conditions. In this study, the multiple driving forces were synergistically used, instead of enhancing lysine biosynthesis, to pump the metabolic flux for glutarate production. This strategy only requires optimized expression of the Cadaverine pathway and the corresponding transporter genes, together with the deletion of one regulator gene. The final strain produced $6.21 \mathrm{~g} \mathrm{~L}^{-1}\left(0.34 \mathrm{~mol} \mathrm{~mol}^{-1}\right)$ of glutarate in shake flasks. However, under fed-batch conditions, the titer reached $54.5 \mathrm{~g} \mathrm{~L}^{-1}$, lower than that obtained by C. glutamicum $\left(90 \mathrm{~g} \mathrm{~L}^{-1}\right)$. It is reported that $C$. glutamicum can grow in the presence of up to $60 \mathrm{~g} \mathrm{~L}^{-1}$ of glutarate ${ }^{12}$, whereas the growth of $E$. coli is severely inhibited at $20 \mathrm{~g} \mathrm{~L}^{-1}$ of glutarate ${ }^{13}$. Therefore, the strain tolerance is a major limitation for glutarate production in $E$. coli, which could be solved by adaptive laboratory evolution.

Cell membrane is selective permeable and transportation of many substances are facilitated by specific transporters. Transporter engineering has been successfully used for co-utilization of mixed carbon sources ${ }^{39}$ and enhancement of product efflux ${ }^{20}$. However, there are few reports on expressing transporters to reduce extracellular accumulation of pathway intermediates. In 
an example, a co-culture system was designed to produce muconic acid from a glucose/xylose mixture and the permease ShiA was expressed in the downstream strain to achieve efficient transport and assimilation of the intermediate 3-dehydroshikimic acid. ${ }^{40}$ Cadaverine and AMV are two intermediates in glutarate biosynthesis. In this study, extracellular accumulation of these two compounds was diminished by screening and expressing specific transporters, which is an essential step toward efficient glutarate production.

\section{Methods}

Experimental materials. Strains and plasmids used in this study are listed in Supplementary Table 1 and Supplementary Table 2, respectively. Primers designed were listed in Supplementary Table 3. E. coli XL-1 Blue was used as the host for plasmid construction. E. coli BW and its derived strains were used for feeding experiment and de novo production. E. coli strain BL21 Star (DE3) was used for protein expression and purification. Plasmids pZE12-luc (high-copy number), pCS27 (medium-copy number), and pSA74 (low-copy number) were used for pathway construction ${ }^{41}$. Plasmid pETDuet-1 was used as the vector for expression and purification of PatA and PatD. Plasmids were constructed by standard enzyme digestion and ligation. Point mutations were introduced by overlap PCR.

BW $\Delta c a d B, \Delta s a p B$, and $\Delta p o t E$ knockout strains were purchased from Coli Genetic Stock Center. The other knockout strains were constructed by RED recombination following the standard protocols ${ }^{42}$.

Culture media and conditions. Luria-Bertani (LB) medium containing yeast extract $\left(5 \mathrm{~g} \mathrm{~L}^{-1}\right)$, tryptone $\left(10 \mathrm{~g} \mathrm{~L}^{-1}\right)$, and $\mathrm{NaCl}\left(10 \mathrm{~g} \mathrm{~L}^{-1}\right)$ was used for inoculant preparation and cell propagation. $\mathrm{M} 9$ medium containing glucose $\left(10 \mathrm{~g} \mathrm{~L}^{-1}\right)$, yeast extract $\left(2 \mathrm{~g} \mathrm{~L}^{-1}\right), \mathrm{NH}_{4} \mathrm{Cl}\left(1 \mathrm{~g} \mathrm{~L}^{-1}\right), \mathrm{Na}_{2} \mathrm{HPO}_{4}\left(6.78 \mathrm{~g} \mathrm{~L}^{-1}\right), \mathrm{KH}_{2} \mathrm{PO}_{4}\left(3 \mathrm{~g} \mathrm{~L}^{-1}\right)$, MOPS (morpholinepropanesulfonic acid, $\left.2 \mathrm{~g} \mathrm{~L}^{-1}\right), \mathrm{NaCl}\left(0.5 \mathrm{~g} \mathrm{~L}^{-1}\right), \mathrm{MgSO}_{4}(1$ $\mathrm{mM})$, and $\mathrm{CaCl}_{2}(0.1 \mathrm{mM})$ was used for feeding experiments. M10 medium containing glucose $\left(25 \mathrm{~g} \mathrm{~L}^{-1}\right)$, yeast extract $\left(2 \mathrm{~g} \mathrm{~L}^{-1}\right),\left(\mathrm{NH}_{4}\right)_{2} \mathrm{SO}_{4}\left(16 \mathrm{~g} \mathrm{~L}^{-1}\right), \mathrm{KH}_{2} \mathrm{PO}_{4}$ $\left(1 \mathrm{~g} \mathrm{~L}^{-1}\right), \mathrm{MgSO}_{4}\left(1 \mathrm{~g} \mathrm{~L}^{-1}\right), \mathrm{CaCO}_{3}\left(30 \mathrm{~g} \mathrm{~L}^{-1}\right)$, and $\mathrm{CaCl}_{2}(0.1 \mathrm{mM})$ was used for de novo production.

For shake flask experiments, single fresh colonies were inoculated into $4 \mathrm{ml}$ of LB media and grown overnight at $37^{\circ} \mathrm{C}$. Subsequently, overnight cultures $(1 \mathrm{ml})$ were transferred to $50 \mathrm{~mL}$ of fresh $\mathrm{M} 9$ or M10 media, grown at $37^{\circ} \mathrm{C}$ and 200 r.p.m. for $2 \mathrm{~h}$ and then induced with $0.5 \mathrm{mM}$ isopropyl- $\beta$-D-thiogalactoside (IPTG). The induced cultures continued to grow at $30^{\circ} \mathrm{C}$ and 200 r.p.m. Ampicillin, kanamycin, and chloramphenicol were added to the medium when necessary at final concentrations of 100,50 , and $34 \mu \mathrm{g} \mathrm{mL}^{-1}$, respectively. Samples were taken at regular time intervals for analysis of cell growth and product accumulation.

For the fed-batch cultivation, strain BM31PE and BM31PER was cultivated in $3 \mathrm{~L}$ bioreactors containing $1 \mathrm{~L}$ of modified M10 medium. The initial medium (per liter) contains the following: glucose (15 g), $\left(\mathrm{NH}_{4}\right)_{2} \mathrm{SO}_{4}(8 \mathrm{~g}), \mathrm{KH}_{2} \mathrm{PO}_{4}(6 \mathrm{~g}), \mathrm{Na}_{2} \mathrm{HPO}_{4}(1$ $\mathrm{g})$, yeast extract $(10 \mathrm{~g}), \mathrm{MgSO}_{4}(2 \mathrm{~g})$, citric acid $(0.8 \mathrm{~g}), \mathrm{V}_{\mathrm{B} 1}(30 \mathrm{mg}), \mathrm{V}_{\mathrm{B} 6}(30 \mathrm{mg})$, and $5 \mathrm{~mL}$ of trace element solution $\left(10 \mathrm{~g} \mathrm{FeSO}_{4} \cdot 7 \mathrm{H}_{2} \mathrm{O}, 2 \mathrm{~g} \mathrm{CaCl}_{2}, 2.2 \mathrm{~g} \mathrm{ZnSO}_{4} \cdot 7 \mathrm{H}_{2} \mathrm{O}, 0.5 \mathrm{~g}\right.$ $\mathrm{MnSO}_{4} \cdot 4 \mathrm{H}_{2} \mathrm{O}, 1 \mathrm{~g} \mathrm{CuSO}_{4} \cdot 5 \mathrm{H}_{2} \mathrm{O}, 0.1 \mathrm{~g}\left(\mathrm{NH}_{4}\right)_{6} \mathrm{Mo}_{7} \mathrm{O}_{24} \cdot 7 \mathrm{H}_{2} \mathrm{O}, 0.23 \mathrm{~g} \mathrm{Na}_{2} \mathrm{~B}_{4} \mathrm{O}_{7} \cdot 10 \mathrm{H}_{2} \mathrm{O}$ per liter). The seed culture was inoculated into the bioreactor at $8 \%$ and cultivated at $37^{\circ} \mathrm{C}$. When $\mathrm{OD}_{600}$ reached around $15,0.5 \mathrm{mM}$ IPTG was added to induce protein expression and then the temperature was shifted to $30^{\circ} \mathrm{C}$. Glucose concentration was maintained below $5 \mathrm{~g} \mathrm{~L}^{-1}$. The feeding solution (per liter) contains glucose $(625 \mathrm{~g})$, $\mathrm{KH}_{2} \mathrm{PO}_{4}(22.5 \mathrm{~g}), \mathrm{Na}_{2} \mathrm{HPO}_{4}(3.75 \mathrm{~g})$, and $\mathrm{MgSO}_{4}(2.5 \mathrm{~g})$. The $\mathrm{pH}$ was maintained at 7.0 during the whole process. The cell growth was monitored by measuring the optical density at $600 \mathrm{~nm}\left(\mathrm{OD}_{600}\right)$ and the supernatants was subjected to high-performance liquid chromatography (HPLC) analysis.

Assays of PatA and PatD. Plasmids pET-patA and pET-patD were transformed into E. coli BL21 Star (DE3), separately. The recombinant strains were cultured to an $\mathrm{OD}_{600}$ of 0.6 and induced with $0.5 \mathrm{mM}$ IPTG for $12 \mathrm{~h}$ at $25^{\circ} \mathrm{C}$. Cells were collected and re-suspended in lysis buffer $(50 \mathrm{mM}$ Tris- $\mathrm{HCl}, 300 \mathrm{mM}$ sodium chloride, $10 \mathrm{mM}$ imidazole, $\mathrm{pH}$ 8.0). The His-tagged proteins were purified using $\mathrm{Ni}^{+}$-affinity chromatography and protein concentration was determined using the bicinchoninic acid (BCA) method

For PatA assay, the reaction system contains $20 \mathrm{mM}$ cadaverine, $20 \mathrm{mM}$ aketoglutarate, and $0.075 \mathrm{mg}$ of purified PatA in $100 \mathrm{mM}$ Tris- $\mathrm{HCl}$ buffer ( $\mathrm{pH} 8.5$ ) with the final volume $1 \mathrm{~mL}$. The reaction was maintained at $30^{\circ} \mathrm{C}$ for $30 \mathrm{~min}$. A coupled assay was used to estimate the activity of PatD. The system contains $4 \mathrm{mM}$ 5-aminopentanal (produced from cadaverine by PatA), $4 \mathrm{mM} \mathrm{NAD}^{+}$, and 0.122 $\mathrm{mg}$ of purified PatD. Reactions were started by the addition of purified enzyme and maintained at $30{ }^{\circ} \mathrm{C}$ for $30 \mathrm{~min}$. The activity of PatA and PatD were determined by measuring the consumption of cadaverine or the production of $\mathrm{AMV}$, respectively.

HPLC analysis of product and intermediates. Lysine, cadaverine, and AMV were analyzed by HPLC (HITACHI) equipped with a reverse-phase Diamonsil C18 column (Diamonsil $5 \mu \mathrm{m}, 250 \times 4.6 \mathrm{~mm}$ ) and UV-VIS detector. Samples were centrifuged at $7700 \times g$ for $10 \mathrm{~min}$. The supernatant was reacted with phenyl isothiocyanate, filtered through $0.22 \mu \mathrm{m}$ film, and used for HPLC analysis. Solvent A was methanol and solvent B was water with $0.1 \%$ formic acid. The column temperature was set at $40^{\circ} \mathrm{C}$. The following gradient was used at a flow rate of $1 \mathrm{~mL} \mathrm{~min}^{-1}: 32 \%$ to $80 \%$ solvent A for $20 \mathrm{~min}, 80 \%$ to $32 \%$ solvent $\mathrm{A}$ for $2 \mathrm{~min}$, and $32 \%$ solvent $\mathrm{A}$ for an additional $5 \mathrm{~min}$. Quantification was based on the peak areas at specific wavelengths $(254 \mathrm{~nm})$. The analysis of glutarate and glucose was performed by HPLC (HITACHI) equipped with an Organic Acid Analysis Column (Amine HPX-87H Ion Exclusion Column, $300 \mathrm{~mm} \times 7.8 \mathrm{~mm}$ ) and refractive index detector. The mobile phase was 5 $\mathrm{mM} \mathrm{H}_{2} \mathrm{SO}_{4}$ at a flow rate of $0.5 \mathrm{~mL} \mathrm{~min}^{-1}$ and the oven temperature was set at $60^{\circ} \mathrm{C}$ The product glutarate was analyzed by ESI-MS and the molecular weight was in accordance with that of the glutarate standard (Supplementary Fig. 7).

\section{Data availability}

Data supporting the findings of this work are available within the paper and its Supplementary Information files. A reporting summary for this Article is available as a Supplementary Information file. The datasets generated and analyzed during the current study are available from the corresponding author upon request. The source data underlying Figs. 2-6 and Supplementary Figs. 1-6 are provided as a Source Data file.

Received: 23 February 2019 Accepted: 3 July 2019

Published online: 26 July 2019

\section{References}

1. Zhang, X. et al. Metabolic evolution of energy-conserving pathways for succinate production in Escherichia coli. Proc. Natl Acad. Sci. USA 106, 20180-20185 (2009)

2. Zhao, M. et al. Metabolic engineering of Escherichia coli for producing adipic acid through the reverse adipate-degradation pathway. Metab. Eng. 47, 254-262 (2018).

3. Mishra, M. K., Varughese, S., Ramamurty, U. \& Desiraju, G. R. Odd-even effect in the elastic modulii of alpha,omega-alkanedicarboxylic acids. J. Am. Chem. Soc. 135, 8121-8124 (2013).

4. Perez-Garcia, F., Jorge, J. M. P., Dreyszas, A., Risse, J. M. \& Wendisch, V. F. Efficient production of the dicarboxylic acid glutarate by Corynebacterium glutamicum via a novel synthetic pathway. Front. Microbiol. 9, 2589 (2018).

5. Zhao, M., Li, G. \& Deng, Y. Engineering Escherichia coli for glutarate production as the C5 platform backbone. Appl. Environ. Microbiol. 84 e00814-e00818 (2018).

6. Wang, J., Wu, Y., Sun, X., Yuan, Q. \& Yan, Y. De novo biosynthesis of glutarate via $\alpha$-keto acid carbon chain extension and decarboxylation pathway in Escherichia coli. ACS Synth. Biol. 6, 1922-1930 (2017).

7. Kim, H. T. et al. Metabolic engineering of Corynebacterium glutamicum for the production of glutaric acid, a C5 dicarboxylic acid platform chemical. Metab. Eng. 51, 99-109 (2019).

8. Revelles, O., Espinosa-Urgel, M., Fuhrer, T., Sauer, U. \& Ramos, J. L. Multiple and interconnected pathways for L-lysine catabolism in Pseudomonas putida KT2440. J. Bacteriol. 187, 7500-7510 (2005).

9. Zhang, M. et al. Increased glutarate production by blocking the glutaryl-CoA dehydrogenation pathway and a catabolic pathway involving L-2hydroxyglutarate. Nat. Commun. 9, 2114 (2018).

10. Wendisch, V. F. Microbial production of amino acids and derived chemicals synthetic biology approaches to strain development. Curr. Opin. Biotechnol. 30, 51-58 (2014)

11. Becker, J., Zelder, O., Hafner, S., Schroder, H. \& Wittmann, C. From zero to hero-design-based systems metabolic engineering of Corynebacterium glutamicum for L-lysine production. Metab. Eng. 13, 159-168 (2011).

12. Rohles, C. M. et al. A bio-based route to the carbon-5 chemical glutaric acid and to bionylon-6, 5 using metabolically engineered Corynebacterium glutamicum. Green Chem. 20, 4662-4674 (2018).

13. Adkins, J., Jordan, J. \& Nielsen, D. R. Engineering Escherichia coli for renewable production of the $5 \square$ carbon polyamide building-blocks 5aminovalerate and glutarate. Biotechnol. Bioeng. 110, 1726-1734 (2013).

14. Schneider, B. L., Hernandez, V. J. \& Reitzer, L. Putrescine catabolism is a metabolic response to several stresses in Escherichia coli. Mol. Microbiol. 88, 537-550 (2013)

15. Shaibe, E., Metzer, E. \& Halpern, Y. S. Control of utilization of L-arginine, Lornithine, agmatine, and putrescine as nitrogen sources in Escherichia coli $\mathrm{K}$ -12. J. Bacteriol. 163, 938-942 (1985).

16. Shen, C. R. et al. Driving forces enable high-titer anaerobic 1-butanol synthesis in Escherichia coli. Appl. Environ. Microbiol. 77, 2905-2915 (2011).

17. Zhu, Y., Eiteman, M. A., DeWitt, K. \& Altman, E. Homolactate fermentation by metabolically engineered Escherichia coli strains. Appl. Environ. Microbiol. 73, 456-464 (2007). 
18. Ohta, K., Beall, D. S., Mejia, J. P., Shanmugam, K. T. \& Ingram, L. O. Genetic improvement of Escherichia coli for ethanol production: chromosomal integration of Zymomonas mobilis genes encoding pyruvate decarboxylase and alcohol dehydrogenase II. Appl. Environ. Microbiol. 57, 893-900 (1991).

19. Lee, S. J. et al. Metabolic engineering of Escherichia coli for enhanced production of succinic acid, based on genome comparison and in silico gene knockout simulation. Appl. Environ. Microbiol. 71, 7880-7887 (2005).

20. Jones, C. M., Lozada, N. J. H. \& Pfleger, B. F. Efflux systems in bacteria and their metabolic engineering applications. Appl. Microbiol. Biotechnol. 99, 9381-9393 (2015).

21. Ajikumar, P. K. et al. Isoprenoid pathway optimization for taxol precursor overproduction in Escherichia coli. Science 330, 70-74 (2010).

22. $\mathrm{Xu}, \mathrm{P}$. et al. Modular optimization of multi-gene pathways for fatty acids production in E. coli. Nat. Commun. 4, 1409 (2013).

23. Lemonnier, M. \& Lane, D. Expression of the second lysine decarboxylase gene of Escherichia coli. Microbiology 144, 751-760 (1998).

24. Jorge, J. M., Leggewie, C. \& Wendisch, V. F. A new metabolic route for the production of gamma-aminobutyric acid by Corynebacterium glutamicum from glucose. Amino Acids 48, 2519-2531 (2016).

25. Schneider, B. L. \& Reitzer, L. Pathway and enzyme redundancy in putrescine catabolism in Escherichia coli. J. Bacteriol. 194, 4080-4088 (2012).

26. Soksawatmaekhin, W., Kuraishi, A., Sakata, K., Kashiwagi, K. \& Igarashi, K. Excretion and uptake of cadaverine by $\mathrm{CadB}$ and its physiological functions in Escherichia coli. Mol. Microbiol. 51, 1401-1412 (2004).

27. Sugiyama, Y. et al. A novel putrescine exporter SapBCDF of Escherichia coli. J. Biol. Chem. 291, 26343-26351 (2016).

28. Kashiwagi, K., Shibuya, S., Tomitori, H., Kuraishi, A. \& Igarashi, K. Excretion and uptake of putrescine by the PotE protein in Escherichia coli. J. Biol. Chem. 272, 6318-6323 (1997).

29. Kashiwagi, K. et al. Isolation of polyamine transport-deficient mutants of Escherichia coli and cloning of the genes for polyamine transport proteins. J. Biol. Chem. 265, 20893-20897 (1990).

30. Pistocchi, R. et al. Characteristics of the operon for a putrescine transport system that maps at 19 minutes on the Escherichia coli chromosome. J. Biol. Chem. 268, 146-152 (1993).

31. Kurihara, S. et al. The putrescine Importer PuuP of Escherichia coli K-12. J. Bacteriol. 191, 2776-2782 (2009).

32. Qian, Z. G., Xia, X. X. \& Lee, S. Y. Metabolic engineering of Escherichia coli for the production of putrescine: a four carbon diamine. Biotechnol. Bioeng. 104, 651-662 (2009).

33. Becker, J., Zelder, O., Häfner, S., Schröder, H. \& Wittmann, C. From zero to hero-design-based systems metabolic engineering of Corynebacterium glutamicum for l-lysine production. Metab. Eng. 13, 159-168 (2011).

34. Yamamoto, K. \& Ishihama, A. Two different modes of transcription repression of the Escherichia coli acetate operon by IclR. Mol. Microbiol. 47, 183-194 (2003).

35. Lin, Y., Sun, X., Yuan, Q. \& Yan, Y. Extending shikimate pathway for the production of muconic acid and its precursor salicylic acid in Escherichia coli. Metab. Eng. 23, 62-69 (2014).

36. Sun, X., Lin, Y., Yuan, Q. \& Yan, Y. Biological production of muconic acid via a prokaryotic 2,3-dihydroxybenzoic acid decarboxylase. ChemSusChem 7, 2478-2481 (2014).

37. Noda, S., Shirai, T., Mori, Y., Oyama, S. \& Kondo, A. Engineering a synthetic pathway for maleate in Escherichia coli. Nat. Commun. 8, 1153 (2017).

38. Kanjee, U. \& Houry, W. A. Mechanisms of acid resistance in Escherichia coli. Annu. Rev. Microbiol. 67, 65-81 (2013).
39. Jojima, T., Omumasaba, C. A., Inui, M. \& Yukawa, H. Sugar transporters in efficient utilization of mixed sugar substrates: current knowledge and outlook. Appl. Microbiol. Biotechnol. 85, 471-480 (2010).

40. Zhang, H., Pereira, B., Li, Z. \& Stephanopoulos, G. Engineering Escherichia coli coculture systems for the production of biochemical products. Proc. Nat Acad. Sci. USA 112, 8266-8271 (2015).

41. Chen, Z., Sun, X., Li, Y., Yan, Y. \& Yuan, Q. Metabolic engineering of Escherichia coli for microbial synthesis of monolignols. Metab. Eng. 39, 102-109 (2017).

42. Datsenko, K. A. \& Wanner, B. L. One-step inactivation of chromosomal genes in Escherichia coli K-12 using PCR products. Proc. Natl Acad. Sci. USA 97, 6640-6645 (2000).

\section{Acknowledgements}

This work was supported by National Key Research and Development Program of China (2018YFA0901800 and 2018YFA0901400) and National Natural Science Foundation of China (21606012, 21636001, and 21776008).

\section{Author contributions}

X.S. and Q.P. designed the experiments. W.L. performed the experiments. L.M., X.S., and J.W. performed the fed-batch production experiment. Q.F. and L.L. performed the enzyme assays. W.L. and X.S. analyzed the data and wrote the manuscript. Q.P., G.Z., and Y.Y. participated in the discussion and revision of the manuscript.

\section{Additional information}

Supplementary Information accompanies this paper at https://doi.org/10.1038/s41467019-11289-4.

Competing interests: The authors declare no competing interests.

Reprints and permission information is available online at http://npg.nature.com/ reprintsandpermissions/

Peer review information: Nature Communications thanks Kazuei Igarashi, David Nielsen, and the other anonymous reviewer(s) for their contribution to the peer review of this work.

Publisher's note: Springer Nature remains neutral with regard to jurisdictional claims in published maps and institutional affiliations.

(c) (i) Open Access This article is licensed under a Creative Commons C. Attribution 4.0 International License, which permits use, sharing, adaptation, distribution and reproduction in any medium or format, as long as you give appropriate credit to the original author(s) and the source, provide a link to the Creative Commons license, and indicate if changes were made. The images or other third party material in this article are included in the article's Creative Commons license, unless indicated otherwise in a credit line to the material. If material is not included in the article's Creative Commons license and your intended use is not permitted by statutory regulation or exceeds the permitted use, you will need to obtain permission directly from the copyright holder. To view a copy of this license, visit http://creativecommons.org/ licenses/by/4.0/.

(C) The Author(s) 2019 